\title{
Distribution des sédiments cohésifs en relation avec la vitesse de la phase solide dans l'estuaire de la Loire.
}

\author{
Martin Sanchez $^{(\mathrm{a}, \mathrm{b})}$, Patricia Grimigni ${ }^{(\mathrm{b})}$, et Yann Delanoë ${ }^{(\mathrm{a})}$ \\ (a) \\ Université de Nantes, 2 rue de la Houssinière, BP 92208, 44322 Nantes \\ (b) \\ U.M.R. 6143, Esplanade de la paix, 14032 Caen.
}

\section{Résumé}

Cette note concerne une approche globale de la vitesse de la phase solide des sédiments cohésifs de l'estuaire de la Loire couvrant la très large gamme de concentrations observée in situ. La vitesse de chute $W$ des matières en suspension est étudiée comme une variable stochastique, il est constaté qu'elle suit une loi de probabilité en accord avec la loi gamma. La vitesse de tassement $V$ de la crème de vase est caractérisée par un scalaire puisque les sédiments à l'intérieur d'une couche de crème de vase descendent sans vitesse différentielle. Enfin, il est montré que la vitesse de chute entravée caractérise une transition entre la vitesse de chute des matières à l'état dispersé et la vitesse de tassement correspondant aux suspensions très concentrées.

\begin{abstract}
$\underline{\text { Abstract }}$
This paper presents a global approach of the distribution of the cohesive sediments in the estuary of the river Loire in terms of the velocity of the solid phase. The study covers the large range of concentrations observed in situ. It deals with the settling velocity of suspended matter, the hindered settling velocity of intermediate concentration suspensions and the settlement velocity of fluid mud. The settling velocity $W$ is studied as a stochastic variable and it is observed that it is gamma distributed. The settlement velocity $V$ is scalar as the sediments in a layer of fluid mud fall with no differential velocity. Finally, the hindered settling velocity is a transition between $W$ and $V$.
\end{abstract}

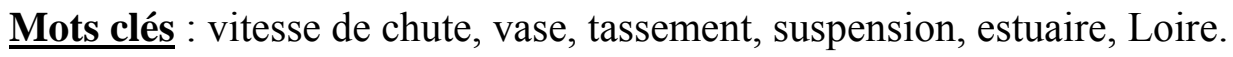

\section{Introduction}

L'estuaire de la Loire (Figure 1) comporte une zone de bouchon vaseux (turbidité maximale) très marquée où la concentration $C$ en matières en suspension MES peut être de 10 à 200 fois celle observée dans le fleuve dans des conditions moyennes qui est de l'ordre de $0.060 \mathrm{~kg} \mathrm{~m}^{-3}$. Les principaux paramètres hydrosédimentaires de cet estuaire sont récapitulés dans le tableau I.

La zone du bouchon vaseux a une étendue moyenne de $20 \mathrm{~km}$, son centre de gravité se situe en fonction du débit fluvial à Nantes en étiage ou à l'embouchure en crue (Migniot et Le Hir, 1994). La figure 2 montre l'emplacement de la crème de vase, du bouchon vaseux et du front de salinité à $0.5 \%$ en fonction $\mathrm{du}$ débit fluvial. 
Session 2: Dynamique sédimentaire et transports de particules

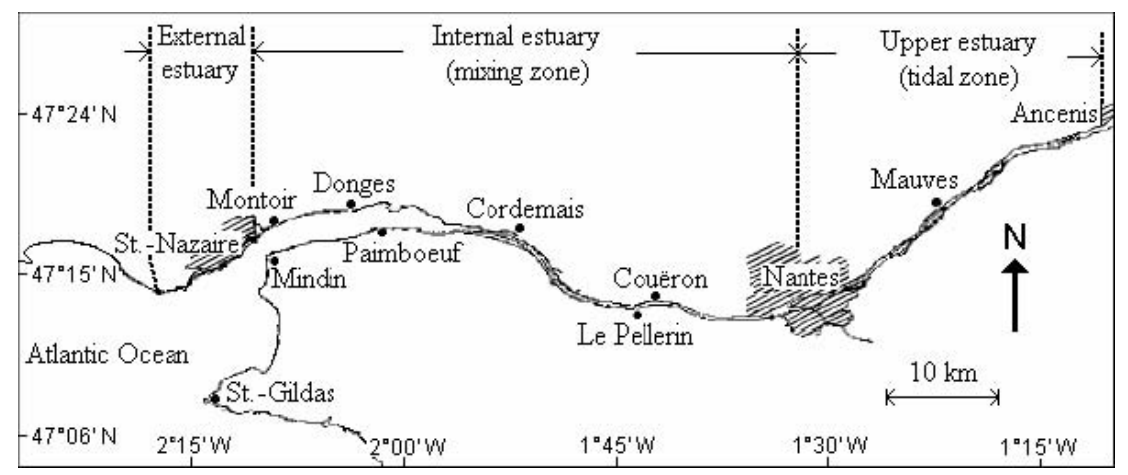

Figure 1 L'estuaire de la Loire (France).

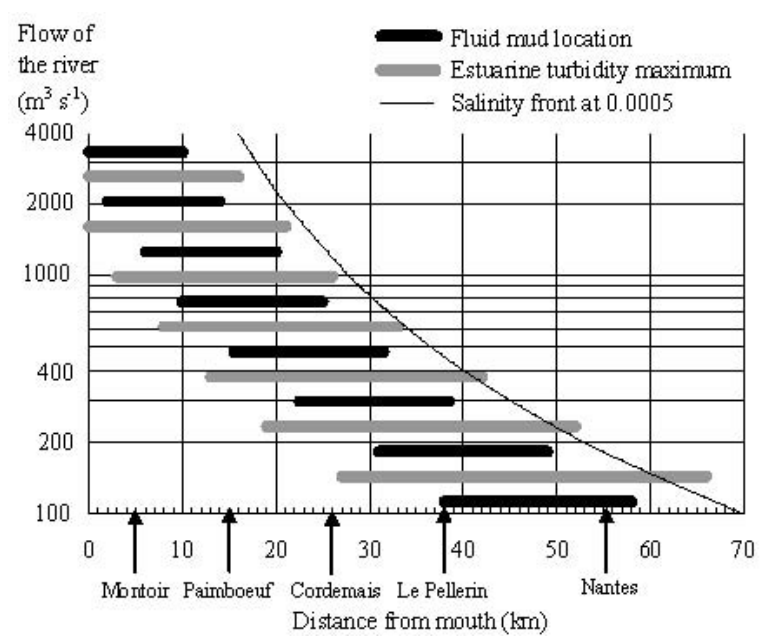

Figure 2 Localisation et extension de la crème de vase (fluid mud) et du bouchon vaseux (estuarine turbidity maximum), et position du front de salinité à 0.5\% (graphique élaboré à partir de donnés publiés par Migniot et Le $\operatorname{Hir}(1994))$.

Tableau I Principaux paramètres hydrosédimentaires de l'estuaire de la Loire.

\begin{tabular}{|cc|cc|}
\hline Paramètre & Valeur & Paramètre & Valeur \\
\hline Débit fluvial moyen & $825 \mathrm{~m}^{3} \mathrm{~s}^{-1}$ & Apports en suspension par le fleuve & $\sim 1.5 \times 10^{9} \mathrm{~kg} \mathrm{an}^{-1}$ \\
Débit fluvial maximum annuel & $5200 \mathrm{~m}^{3} \mathrm{~s}^{-1}$ & Expulsion de MES vers l'océan & $\sim 0.5 \times 10^{9} \mathrm{~kg} \mathrm{an}^{-1}$ \\
Débit fluvial minimum annuel & $77 \mathrm{~m} \mathrm{~s}^{-1}$ & Taux d'envasement résiduel & $\sim 1.0 \times 10^{9} \mathrm{~kg} \mathrm{an}^{-1}$ \\
Marnage maximum à l'embouchure & $6.40 \mathrm{~m}^{-1}$ & Dragages d'entretien moyens & $\sim 3.3 \times 10^{6} \mathrm{~m}^{-1} \mathrm{an}^{-1}$ \\
Prisme de marée en vive-eau & $2.5 \times 10^{8} \mathrm{~m}^{3}$ & Longueur moyenne de la crème de vase & $\sim 12.5 \mathrm{~km}$ \\
Pénétration max de la marée dynamique & $100 \mathrm{~km}$ & Longueur moyenne du bouchon vaseux & $\sim 20 \mathrm{~km}$ \\
Pénétration max de la marée saline & $70 \mathrm{~km}$ & Stock moyen de vase "mobile" & $\sim 1.0 \times 10^{9} \mathrm{~kg}$ \\
\hline
\end{tabular}

Une accumulation importante de vase "mobile" représentant dans l'estuaire de la Loire environ 1×109 kg de sédiments (CSEEL, 1984 ; Delft Hydraulics, 1994 ; Fera, 1994), soit l'équivalent de 8 mois d'apport par le fleuve dans des conditions moyennes, est présente dans la zone de turbidité maximale. 
Au cours d'un cycle semi-lunaire de variation du coefficient de marée, le stock de vase "mobile" se présente dans l'estuaire sous deux formes différentes (Gallenne, 1974) : en morteseaux la plus grande partie de ces matériaux s'accumule au fond sous la forme de crème de vase et seulement une petite partie de cette crème de vase est remise momentanément en suspension au cours d'un cycle de marée, tandis qu'en vives-eaux (coefficient de marée supérieur à 75), une grande partie de la crème de vase est remise en suspension entraînant la formation d'un bouchon vaseux, et seule la fraction de crème de vase dont la concentration s'est accrue suffisamment à la suite du tassement contribue à un envasement résiduel de l'estuaire.

Dans la zone de turbidité maximale, on rencontre des mélanges vase-eau dont la concentration varie dans une très large gamme de valeurs. Selon leurs concentrations, ces mélanges sont soumis aux processus sédimentaires suivants :

a) chute et décantation des matières en suspension ;

b) tassement des suspensions fortement concentrées ;

c) consolidation des dépôts de vase.

Les états des complexes vase-eau associés aux processus précédents sont :

a) suspension diluée $\left(C<15 \mathrm{~kg} \mathrm{~m}^{-3}\right)$;

b) suspension fortement concentrée ou crème de vase $\left(40 \mathrm{~kg} \mathrm{~m}^{-3}<C<325 \mathrm{~kg} \mathrm{~m}_{-3}^{-3}\right)$;

c) dépôts de vases rigides pourvues de contraintes effectives $(C>325 \mathrm{~kg} \mathrm{~m})$.

Quel que soit le processus sédimentaire considéré, le principal paramètre affectant la dynamique verticale des mixtures vase-eau est la vitesse de la phase solide sous l'effet de la pesanteur. En fonction des états cités ci-dessus, ces vitesses sont désignées comme suit :

a) vitesse de chute $W$ des matières en suspension qui dépend de la taille, de la forme et de la masse des agrégats en suspension ;

b) vitesse de tassement $V$ qui dépend de la perméabilité de la crème de vase ;

c) vitesse de la phase solide liée à la consolidation qui dépend de la perméabilité et de l'état des contraintes effectives dans le dépôt de vase.

Dans ce manuscrit on s'intéresse particulièrement à la description de deux phénomènes affectant la dynamique verticale du stock de vase "mobile" : la chute des matières en suspension et le tassement de la crème de vase.

Des mesures en laboratoire et in situ ont montré que la vitesse de chute $W$ des MES (Krone, 1962 ; Thorn, 1981 ; Migniot, 1989 ; Wolanski et al., 1992 ; Orton et Kineke, 2001 ; Tattersall et al., 2003) et la vitesse de tassement $V$ des crèmes de vase (Kynch, 1952 ; Been, 1980 ; Tan et al., 1990 ; Sanchez, 1992 ; Sanchez et Grovel, 1994b ; Wett, 2002) varient principalement avec la concentration suivant des lois propres à chaque sédiment.

L'étude en laboratoire des sédiments reconstitués en suspension montre que la vitesse de chute $W$ dépend également du cisaillement turbulent dans la colonne d'eau (Manning et Dyer, 1999). Cependant, aucune mise en évidence de cette relation n'a été possible dans une zone côtière soumise à l'action de la marée (Lumborg et Windelin, 2003).

D'une part, la vitesse de chute $W$ des vases de Loire augmente progressivement avec la concentration dans le domaine des concentrations inférieures à $10 \mathrm{~kg} \mathrm{~m}^{-3}$ (Sanchez, 1992 ; Sanchez et al., 2004). D'autre part, pour les concentrations supérieures à environ $40 \mathrm{~kg} \mathrm{~m}^{-3}$, la vitesse de tassement $V$ diminue lorsque $C$ augmente (Sanchez, 2000). Une différence importante entre la chute des MES et le tassement de la crème de vase est que la décantation des MES provoque une ségrégation des agrégats en fonction de leurs vitesses de chute $W$, alors que 
pendant le tassement, la vitesse de la phase solide $V$ est la même pour tous les sédiments adjacents situés au même niveau sur la verticale (Wett, 2002).

\section{$\underline{\text { 2.Vitesse de chute des matières en suspension }}$}

La plupart des auteurs caractérise la vitesse de chute par sa valeur médiane qui est toujours inférieure à la vitesse de chute moyenne $\bar{W}$, laquelle donne une meilleure description de la dynamique verticale des sédiments. Usuellement dans les modèles hydrosédimentaires la vitesse de chute est représentée par un scalaire dont la valeur dépend de $C$. Cependant, compte tenu du grand nombre d'agrégats divers se trouvant simultanément en suspension, la vitesse de chute $W$ est en réalité une variable stochastique.

La distribution des matières en suspension en termes des vitesses de chute a été étudiée par Sanchez et al. (2004) avec la méthode du tube d'Owen (1976). Cette étude montre que la vitesse de chute moyenne $\overline{W_{0}}\left(\mathrm{~m} \mathrm{~s}^{-1}\right)$ des sédiments en suspension près du fond peut être rattachée à la concentration $C_{0}\left(\mathrm{~kg} \mathrm{~m}^{-3}\right)$ de référence au fond (en $\left.\mathrm{z}=0\right)$ par :

$$
\begin{array}{lll}
\overline{W_{0}}=0.001 C_{0}{ }^{0.50} & \text {,pour : } & C_{0}<10 \mathrm{~kg} \mathrm{~m}^{-3} \\
\overline{W_{0}}=0.00316 & \text {,pour : } & 10 \mathrm{~kg} \mathrm{~m}^{-3}<C_{0}<15 \mathrm{~kg} \mathrm{~m}^{-3}
\end{array}
$$

Il a été observé (Sanchez et al., 2004) que la vitesse de chute $W$ des MES au fond suit toujours une distribution en accord avec la loi de probabilité gamma. Ainsi, la fonction de densité de probabilité fdp $f(w)$ de $W$ pour les MES au fond est bien décrite par l'expression suivante :

$$
f(w)=\frac{\lambda_{0}{ }^{r} w^{r-1} \exp \left(-\lambda_{0} w\right)}{\Gamma(r)}, 0 \leq w<\infty
$$

où $\Gamma$ est la fonction gamma, $\lambda_{0}=r \bar{W}$ un paramètre, et $r={\overline{W_{0}}}^{2} / \sigma\left[W_{0}\right]^{2}$ (avec $\sigma\left[W_{0}\right]$ désignant l'écart-type des valeurs de $W$ pour les sédiments du fond) un deuxième paramètre de la fdp qui caractérise l'étendue relative des valeurs de la vitesse de chute. Ce paramètre est également rattaché à $C_{0}\left(\mathrm{~kg} \mathrm{~m}^{-3}\right)$ par :

$$
\begin{array}{lll}
r=0.80 C_{0}^{0.34} & \text {,pour : } C_{0}<10 \mathrm{kgm}^{-3} \\
r=1.75 & \text {,pour: } 10 \mathrm{kgm}^{-3}<C_{0}<15 \mathrm{kgm}^{-3}
\end{array}
$$

La figure 3 montre la distribution cumulée des matières en suspension au fond en accord avec la fonction de densité de probabilité définie par l'équation 2, pour plusieurs valeurs de la concentration au fond $C_{0}\left(\mathrm{~kg} \mathrm{~m}^{-3}\right)$. Cette distribution cumulée représente la quantité des matières en suspension en $\mathrm{kg} \mathrm{m}$ ayant des vitesses de chute $W$ inférieurs à $w$.

En accord avec la dynamique verticale distributive des sédiments, la vitesse de chute $W$ obéit à la loi de probabilité gamma à chaque niveau $z$, avec le paramètre $r$ restant invariant. De plus, la variation verticale de la vitesse de chute moyenne locale $\bar{W}$ en fonction de la concentration locale $C$ est régie par:

$$
\bar{W}=\overline{W_{0}}\left(\frac{C}{C_{0}}\right)^{1 / r}
$$


Il faut remarquer qu'une distribution de la vitesse de chute $W$ en accord avec la loi de probabilité gamma est compatible avec les spectres de taille («size spectra ») définis par Kranck et Milligan (1992).

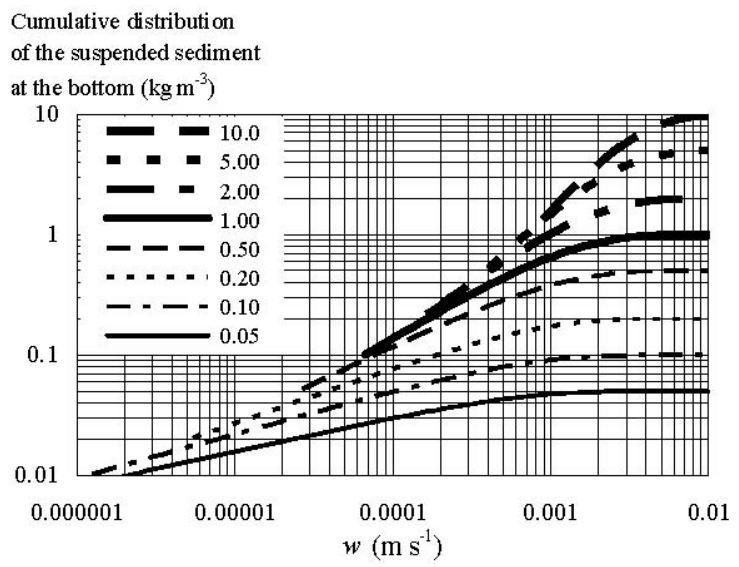

Figure 3 Distribution cumulée des matières en suspension au fond en accord avec la fonction de densité de probabilité définie par l'équation 2, pour plusieurs valeurs de la concentration au fond $C_{0}\left(\mathrm{~kg} \mathrm{~m}^{-3}\right)$.

\section{$\underline{\text { 3.Tassement de la crème de vase }}$}

Au dessus d'un fond imperméable, la vitesse de tassement (vitesse de la phase solide) à l'intérieur d'une couche de crème de vase dépourvue de contraintes effectives est décrite par l'équation de Been (1980):

$$
V=\frac{k}{\rho_{w}}\left(1-\frac{\rho_{w}}{\rho_{s}}\right) C
$$

où $k$ est le coefficient de perméabilité qui dépend exclusivement de la concentration $C, \rho_{s}$ la masse volumique de la phase solide $\left(\rho_{s} \approx 2550 \mathrm{~kg} \mathrm{~m}^{-3}\right)$ et $\rho_{w}$ la masse volumique de la phase fluide. Pour un grand nombre de matériaux, la variation de $k$ avec $C$ s'accorde avec la loi suivante (Sanchez et Grovel, 1993) :

$$
k=A_{1} \exp \left[-A_{2}\left(\frac{C}{\rho_{s}}\right)^{A_{3}}\right]
$$

où $A 1$ (m s-1), $A_{2}$ et $A_{3}$ sont des constantes propres à chaque matériau. Dans un domaine étroit de valeurs de $C$ la valeur de $A 3$ est proche de 1 (Sanchez et Grovel, 1993), cependant, lorsque le domaine de variation de $C$ est large sa valeur est inférieure à 1 .

Des valeurs de $k$ en fonction de $C$ peuvent être évaluées à partir des courbes de tassement établies en laboratoire avec des essais en colonne de tassement grâce à une méthode graphique développée par Kynch (1952), modifiée par Tan et al. (1990) et par Sanchez et Grovel (1994a). La figure 4 montre pour la vase de Loire un certain nombre de valeurs de $k$ en fonction de $C$ évaluées à partir des mesures effectuées par plusieurs auteurs (Migniot, 1989 ; Sanchez, 1992 ; Le Borgne, 1992 ; Ouraq, 1996 ; Sanchez, 2000) et au cours de cette étude (Grimigni, 2003). 


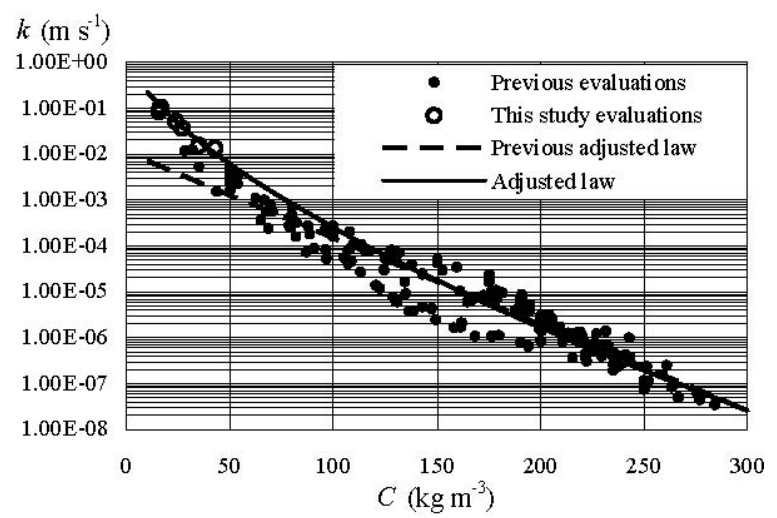

Figure 4 Variation du coefficient de perméabilité $k$ en fonction de la concentration C, pour la vase de Loire.

L'ajustement de l'équation 6 pour la vase de Loire permet l'évaluation de $A_{1}=1.60 \mathrm{~m} \mathrm{~s}-1$, $A_{2}=72$, et $A_{3}=0.65$, dans le domaine $15 \mathrm{~kg} \mathrm{~m}^{-3}<C<325 \mathrm{~kg} \mathrm{~m}^{-3}$.

Le débit solide $Q_{C}$ à travers une interface de concentration $C$ constante dans le temps à l'intérieur d'une crème de vase en cours de tassement (c'est-à-dire, la quantité de masse solide qui excède la concentration $C$ par unité de surface horizontale et par unité de temps) est donnée par (Sanchez et Grovel, 1993) :

$$
Q_{C}=V C\left[A_{2} A_{3}\left(\frac{C}{\rho_{s}}\right)^{A_{3}}-1\right]
$$

La figure 5 montre $Q=V C$ et $Q_{C}$ en fonction de $C$. On y observe que la concentration $C_{\min }$ correspondant au début du phénomène de tassement est proche de $40 \mathrm{~kg} \mathrm{~m}^{-3}$. Cette concentration est associée au maximum de $Q_{C}$.

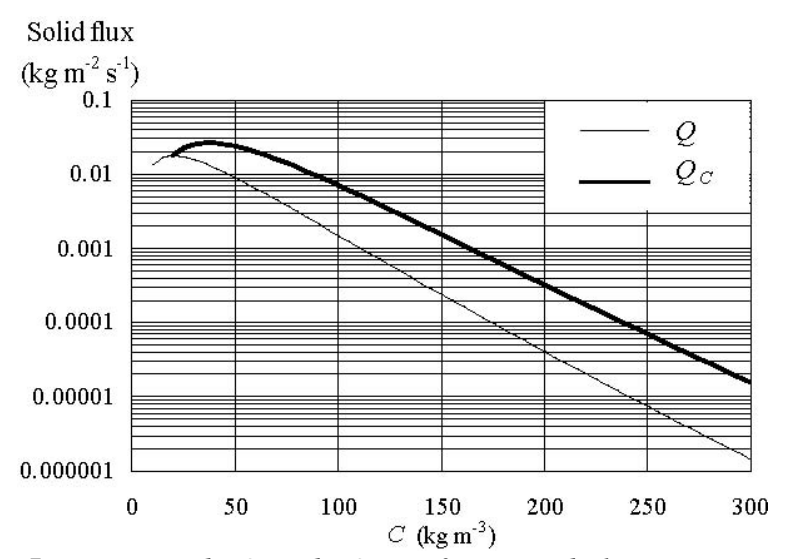

Figure 5 Variation de $Q$ et de $Q_{C}$ en fonction de la concentration $C$. 


\section{Vitesse de chute entravée des matières en suspension}

La vitesse de chute entravée correspond à un domaine de concentrations variant de $15 \mathrm{~kg} \mathrm{~m}$ à $40 \mathrm{~kg} \mathrm{~m}^{-3}$. La vitesse de la phase solide subit une transition entre la vitesse de chute des matières en suspension et la vitesse de tassement des vases à l'état de crème. Dans ce domaine on observe une diminution de la vitesse descendante de la phase solide lorsque la concentration augmente. Ce phénomène est expliqué par Mehta (1986) par le fait que les agrégats de vase forment un réseau continu tridimensionnel impliquant un écoulement ascendant de l'eau à travers les interstices afin que la phase solide puisse descendre.

Des mesures en laboratoire effectuées par Grimigni (2003) ont montré que dans le domaine $15 \mathrm{~kg} \mathrm{~m}^{-3}<C<40 \mathrm{~kg} \mathrm{~m}^{-3}$, la vitesse de la phase solide obéit à deux lois distinctes :

a) les agrégats dont la vitesse de chute $W$ à l'état dispersé est supérieure à $V$ (équation 5), tombent en bloc avec cette vitesse $V$;

b) les autres agrégats plus légers tombent plus lentement avec leurs vitesses de chute individuelles à l'état dispersé.

Dans ce domaine des concentrations, la vitesse de chute entravée obéit à la fonction de répartition suivante (Figure 6) :

$$
\begin{array}{ll}
F(w)=\int_{0}^{V} \frac{\Lambda_{0}^{r_{\max }} w^{r_{\max }-1} \exp \left(-\Lambda_{0} w\right)}{\Gamma\left(r_{\max }\right)} d w & \text {,pour : } w<V \\
F(w)=1 & \text {,pour : } w \geq V
\end{array}
$$

où $V$ est la vitesse de tassement donnée par l'équation de Been (équation 5), et $\Lambda_{0}=r_{\max } /{ }^{W_{0 \max }}$ est la valeur de $\lambda_{0}$ associée à $\overline{W_{0}}=\overline{W_{0 \max }}$ et à $r=r_{\max }$, avec $\overline{W_{0 \max }}$ et $r_{\max }$ désignant respectivement les maxima de $\frac{0}{W_{0}}$ et de $r$ observés pour une concentration égale à $10 \mathrm{~kg} \mathrm{~m}^{-3}$.

Ainsi, la vitesse de chute entravée pour les agrégats dont la vitesse de chute $W$ à l'état dispersé est supérieure à $V$, peut être décrite par l'équation de Been pour la vitesse de tassement $V$ (équation 5). Dans cette équation, la diminution de la vitesse observée quand la concentration augmente est due principalement à la réduction du coefficient de perméabilité (ou conductivité hydraulique) $k$.

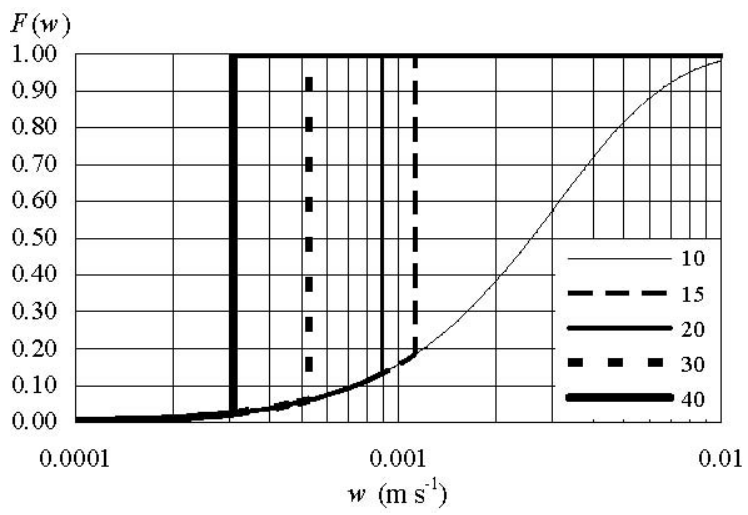

Figure 6 Fonction de répartition de la vitesse de la phase solide pour plusieurs valeurs de la concentration dans le domaine $10 \mathrm{~kg} \mathrm{~m}^{-3}<\mathrm{C}<40 \mathrm{~kg} \mathrm{~m}^{-3}$. 


\section{Conclusions}

Cette note présente une approche globale caractérisant la vitesse de la phase solide des sédiments cohésifs de l'estuaire de la Loire couvrant la très large gamme de concentration $C$ observée in situ.

Pour les matières en suspension diluées $\left(0.02 \mathrm{~kg} \mathrm{~m}^{-3}<C<15 \mathrm{~kg} \mathrm{~m}^{-3}\right)$ la distribution de la vitesse de chute $W$ de la masse sédimentaire obéit à une loi gamma comportant un paramètre $r$ qui caractérise l'étendue des valeurs de la vitesse de chute des différents agrégats simultanément en suspension. La variation verticale de la vitesse de chute moyenne locale est proportionnelle à la puissance $1 / r$ de la concentration locale $C$. Ceci est le résultat de la dynamique verticale affectant les matières en suspension selon leur vitesse de chute $W$.

Pour les applications pratiques, la vitesse de chute moyenne $\overline{W_{0}}$ près du fond est reliée à la concentration de référence au fond $C_{0}$ (équation 1). La variation verticale de la vitesse de chute moyenne locale $\bar{W}$ est reliée à la concentration locale $C$ à travers l'équation 4 , avec le paramètre $r$ rattaché à $C_{0}$ par l'équation 3. Ceci est équivalent à considérer séparément l'ensemble d'agrégats en suspension selon leurs vitesses de chute respectives, avec une simplification considérable des modélisations. En d'autres termes, quand la vitesse de chute $W$ est distribuée en accord avec la loi gamma, la vitesse de chute moyenne et le paramètre $r$ de la distribution suffisent pour décrire la dynamique verticale de l'ensemble des agrégats en suspension.

Pour la boue fluide ou crème de vase $(40 \mathrm{~kg} \mathrm{~m}<C<300 \mathrm{~kg} \mathrm{~m})$ la phase solide se tasse sans dispersion de matières. La vitesse de tassement $V$ diminue avec l'augmentation de la concentration à cause de la réduction du coefficient de perméabilité $k$. La vitesse de la phase solide peut être modélisée en fonction de $k$ grâce à une loi ajustée pour les sédiments de l'estuaire de la Loire.

Pour les suspensions de concentration intermédiaire $\left(15 \mathrm{~kg} \mathrm{~m}^{-3}<C<40 \mathrm{~kg} \mathrm{~m}^{-3}\right)$ la phase solide est caractérisée par une vitesse de chute entravée. Sur la base d'observations en laboratoire, pour un pourcentage de matières cette vitesse est modélisée par l'approche correspondant aux matières en suspension (vitesse différentielle entre les agrégats en suspension). Pour le pourcentage complémentaire la vitesse est décrite par l'approche correspondant au tassement (chute en bloc de l'ensemble des particules sans vitesse différentielle).

L'ensemble de ces résultats est issu de mesures en laboratoire employant des méthodes adaptées selon l'état du mélange eau-sédiment :

- mesures par la méthode du tube d'Owen pour les matières en suspension,

- mesures par densimétrie pour les suspensions moyennement concentrées,

- mesures en colonne de tassement pour la crème de vase.

Les résultats obtenus montrent l'existence d'une continuité dans les phénomènes de décantation et de tassement, alors que les profils verticaux de la concentration peuvent présenter de discontinuités dans certains cas. 


\section{Références}

1.Been K. (1980). Stress-strain behavior of a cohesive soil deposited under water (257 p.). PhD Thesis, University of Oxford, Oxford, U. K.

2.CSEEL (1984). Rapport final du comité scientifique pour l'environnement de l'estuaire de la Loire (158 p.). Rapports Scientifiques et Techniques $n^{\circ}$ 55, Publications du CNEXO, Brest.

3.Delft Hydraulics (1994). Etude hydrologique de l'estuaire de la Loire (98 p.). Delft hydraulics, Delft, The Netherlands.

4.Fera P. 1994. Estuaire de la Loire (21 p.). Agence de l'eau Loire Bretagne, Nantes.

5.Gallenne B. (1974). Les accumulations turbides dans l'estuaire de la Loire - Etude de la "crème de vase". Thèse, Université de Nantes, 323 p.

6. Grimigni P. (2003). Effet de la stratification des matières en suspension sur la vitesse de chute des sédiments cohésifs (59 p.). DEA, Université de Caen.

7.Kranck, K., et Milligan, T.G. (1992). Characteristics of suspended particles at an 11-hour anchor station in San Francisco Bay, California. Journal of Geophysical Research 97, 1137311382.

8.Krone R.B. (1984). The significance of aggregate properties to transport processes, Estuarine Cohesive Sediment Dynamics ed. by Mehta, Springer-Verlag, 66-84.

9.Kynch G.J. (1952). A theory of sedimentation. Faraday Society Transactions 48, 166-176.

10.Le Borgne M.A. (1992). Optimisation d'une darse en estuaire pour une sédimentation minimale (89 p.). DEA, Université de Nantes.

11.Lumborg, U., et Windelin, A. (2003). Hydrography and cohesive sediment modelling: application to Rømø Dyb tidal area. Journal of Marine Systems 38, 287-303.

12.Manning, A.J., et Dyer, K.R. (1999). A laboratory examination of floc characteristics with regard to turbulent shearing. Marine Geology 160, 147-170.

13.Mehta A.J. (1986). Characterization of cohesive sediment properties and transport processes in estuaries. In A. J. Mehta (Ed.), Estuarine cohesive sediment dynamics, Coastal and estuarine studies $n^{\circ} 14$ (pp. 290-325). Berlin: Springer.

14. Migniot C. (1989). Tassement et rhéologie des vases. La Houille Blanche, $\mathrm{n}^{\circ} 1$ et 2, 11-29 et $95-111$.

15.Migniot C., et Le Hir P. (1994). Estuaire de la Loire : Hydrosédimentaire (84 p.). Rapports de synthèse de l'APEEL $\mathrm{n}^{\circ} 1$, Publication de l'association pour la protection de l'environnement de l'estuaire de la Loire, Nantes.

16.Orton P.M., et Kineke G.C. (2001). Comparing calculated and observed vertical suspended-sediment distributions from a Hudson river estuary turbidity maximum. Estuarine, Coastal and Shelf Science 52, 401-410.

17. Ouraq Y. (1996). Contribution à l'étude des sédiments dans l'environnement estuarien : cas de l'estuaire de la Loire. Thèse, Université de Nantes, 169 p.

18. Owen M.W. (1976). Determination of the settling velocities of cohesive muds. Report No IT 161, Hydraulics Research Station Wallingford, UK.

19. Sanchez M. (1992). Modélisation dans un estuaire à marée - Rôle du bouchon vaseux dans la tenue des sols sous-marins. Thèse, Université de Nantes, $210 \mathrm{p}$.

20.Sanchez M. (2000). Dynamique des sédiments fins en milieu marin et agitation portuaire. mémoire HDR, Université de Caen, $82 \mathrm{p}$.

21.Sanchez M., et Grovel A. (1993). Modélisation du tassement sous poids propre des couches de vase molle et saturée sur un sol imperméable, La Houille Blanche 1, 29-34.

22. Sanchez M., et Grovel A. (1994a). Le tassement des vases comme processus sédimentaire. 
III ${ }^{\text {èmes }}$ Journées Nationales Génie Civil-Génie Côtier, Sète, 69-73.

23.Sanchez M., et Grovel A. (1994b). Settlement: a sedimentary process. In M. Bélorgey, R.D. Rajaona, and J.F.A. Sleath, (Eds.), Euromech 310 Sediment transport mechanisms in coastal environments and rivers (pp. 146-151). Singapore: World Scientific.

24.Sanchez M., Grimigni, P., et Delanoë Y. (2004). Steady-state vertical distribution of the cohesive sediments in a flow. (soumis à une revue internationale).

25.Tan T.S., Yong K.Y., Leong E.C., et Lee S.L. (1990). Sedimentation of clayey slurry. Journal of Geotechnical Engineering 116-6, 885-898.

26.Tattersall G.R., Elliott A.J., et Lynn N.M. (2003). Suspended sediment concentrations in the Tamar estuary. Estuarine, Coastal and Shelf Science 57, 679-688.

27.Thorn M.F.C. (1981). Physical processes of siltation in tidal channels. Proc. Hydraulic Modelling Applied to Maritime Eng. Problems, ICE, London, 47-75.

28. Wett B. (2002). A straight interpretation of the solids flux theory for a three-layer sedimentation model. Water Research 36, 2949-2958.

29.Wolanski E., Gibbs R.J., Mazda Y., Mehta A.J., et King B. (1992). The role of turbulence in settling of mudflocs. Journal of Coastal Research 8 (1), 35-46. 